TITLE:

\title{
Molecular weight dependence of mean square displacement in ultrathin polymer films as studied by inelastic neutron scattering
}

\section{AUTHOR(S):}

Inoue, Rintaro; Kanaya, Toshiji; Nishida, Koji;

Tsukushi, Itaru; Shibata, Kaoru

\section{CITATION:}

Inoue, Rintaro ...[et al]. Molecular weight dependence of mean square displacement in ultrathin polymer films as studied by inelastic neutron scattering. PHYSICAL REVIEW E 2008, 77(3(Part 1)): 032801.

ISSUE DATE:

2008-03

URL:

http://hdl.handle.net/2433/84644

RIGHT:

(c) 2008 The American Physical Society 


\title{
Molecular weight dependence of mean square displacement in ultrathin polymer films as studied by inelastic neutron scattering
}

\author{
Rintaro Inoue, ${ }^{1}$ Toshiji Kanaya, ${ }^{1, *}$ Koji Nishida, ${ }^{1}$ Itaru Tsukushi, ${ }^{2}$ and Kaoru Shibata ${ }^{3}$ \\ ${ }^{1}$ Institute for Chemical Research, Kyoto University, Uji, Kyoto-fu 611-0011, Japan \\ ${ }^{2}$ Chiba Institute of Technology, Narashino, Chiba-ken 275-0023, Japan \\ ${ }^{3}$ Japan Atomic Energy Agency, Tokai, Ibaraki-ken 319-1195, Japan
}

(Received 6 August 2007; published 14 March 2008)

\begin{abstract}
In previous papers, we found that the mean square displacement $\left\langle u^{2}\right\rangle$ in polystyrene thin films decreased with film thickness and discussed two possible causes for the decrease. One is the hard interface layer between the polymer thin film and the substrate, and the other is the spatial confinement effect of polymer chains. In order to disclose the cause of the decrease of $\left\langle u^{2}\right\rangle$ with film thickness, we studied the molecular weight $M_{w}$ dependence of $\left\langle u^{2}\right\rangle$ in polystyrene thin films. We found that $\left\langle u^{2}\right\rangle$ was independent of $M_{w}$ within experimental error, concluding that the interface effect is a main reason for the decrease of $\left\langle u^{2}\right\rangle$ with film thickness.
\end{abstract}

DOI: 10.1103/PhysRevE.77.032801

PACS number(s): 83.80.Sg, 68.60.-p, 61.05.fg, 63.50.-x

\section{INTRODUCTION}

It was reported that the thermal and mechanical properties of polymer thin films are very different from those of the bulk state. Among them, the glass transition temperature $T_{g}$ of thin films is one of the attracting phenomena and the decrease of $T_{g}$ was observed with film thickness for polystyrene [1-4]. It was assumed that a decrease of $T_{g}$ was caused by a mobile surface layer; however, a definite mechanism of the glass transition of thin films is still unknown. The glass transition is a relaxational process; therefore, dynamical studies are needed to understand this phenomenon. Many dynamical studies of polymer thin films were performed using various methods like dielectric relaxation [5-7], atomic force microscopy [8-11], x-ray photon correlation spectroscopy (XPCS) [12], dynamic mechanical analysis [13], inelastic neutron scattering [14-18], dynamic light scattering [19], and so on. We also studied the glassy dynamics of polystyrene thin films with inelastic neutron scattering $[17,18]$ to observe the decrease of mean square displacement $\left\langle u^{2}\right\rangle$ as well as the inelastic and quasielastic neutron scattering intensity with film thickness. We considered two main possibilities for the reason of decrease of $\left\langle u^{2}\right\rangle$ with film thickness. One is the hardening of polymer chains due to the spatial confinement (confinement effect). When the film thickness decreased to less than a polymer chain coil size that is comparable to twice the radius of gyration $\left(2 R_{g}\right)$, polymer chains cannot sustain a normal coil form and the deformation of polymer coils would occur. Under this condition, the deformed polymer chains would have a higher restoring force than the normal coils and result in a decrease of mobility. However, a decrease of mobility $\left(=\left\langle u^{2}\right\rangle\right)$ was observed even for $1000 \AA$ thin film, which is much larger than $2 R_{g}$ in bulk $[17,18]$. In order to understand this phenomenon, we assumed that a hard layer (lower mobility layer) would exist at the interface between the polymer thin film and the substrate. With decreasing film thickness, the contribution from such a

\footnotetext{
*Corresponding author; FAX: +81-774-38-3146; kanaya@scl.kyoto-u.ac.jp
}

hard layer becomes large under the assumption that the thickness of such a hard layer is independent of the film thickness. This is another possibility (interface effect). Except for the two possibilities, we considered other minor possible reasons for the decrease of $\left\langle u^{2}\right\rangle$. One is the end group effect. According to the experiment by Satomi et al. [11] it was negligible for $M_{w}$ above $10^{5}$, but not for $M_{w}$ below $10^{5}$. The other is the surface roughness of polymer thin films. Miyazaki et al. investigated the surface roughness of polystyrene thin films for different $M_{w}$ by x-ray reflectivity (XR) in a wide thickness range [20]; however, the observed roughness was less than $7 \AA$ and not dependent on thickness or $M_{w}$. Therefore, this is negligible too.

In the former paper, analyzing the inelastic data we concluded that the interface effect was a candidate for a decrease of $\left\langle u^{2}\right\rangle$ with film thickness $[17,18]$. However, it was not enough for the final conclusion. In order to settle this problem, we prepared thin films with the same film thickness for different molecular weights $\left(M_{w}\right)$, giving different ratios of the film thickness $(=d)$ to the double radius of gyration $\left(2 R_{g}\right)$. If the confinement effect were dominant, the decrease of $\left\langle u^{2}\right\rangle$ would be observed with the decrease of ratio of $d / 2 R_{g}$ because decreasing the ratio $d / 2 R_{g}$ means an increase of spatial confinement. And if the interface effect were dominant, $\left\langle u^{2}\right\rangle$ would be constant regardless of the ratio $d / 2 R_{g}$ because the ratio is constant under the same film thickness. Conducting the experiment of the $M_{w}$ effect, we can understand which factor is responsible for the decrease of $\left\langle u^{2}\right\rangle$ with film thickness.

\section{EXPERIMENT}

We used three amorphous polystyrenes (PSs) with different molecular weights $\left(M_{w}\right) 2.90 \times 10^{5}, 1.05 \times 10^{6}$, and $1.88 \times 10^{6}$ and the molecular weight distributions $\left(M_{w} / M_{n}\right)$ $1.06,1.07$, and 1.13 , respectively, where $M_{w}$ and $M_{n}$ are the weight average and the number average of the molecular weight, respectively; and calculated values of $R_{g}$ are 147, 280 , and $374 \AA$ for $M_{w}=2.90 \times 10^{5}, 1.05 \times 10^{6}$, and 1.88 $\times 10^{6}$, respectively, under the unperturbed chain approximation [21]. 
PS thin films were prepared by spin-coating toluene solutions at $2000 \mathrm{rpm}$ on flat glass plates rinsed in toluene prior to the spin coating. The film thickness was controlled by varying the concentration of PS in solution, and we prepared films $400 \AA$ in thickness for three different $M_{w}$ 's. This film thickness is less than $2 R_{g}$ for $M_{w}=1.05 \times 10^{6}$ and 1.88 $\times 10^{6}$. The film was removed from the glass plate onto a water surface, then collected on Al foil $15 \mu \mathrm{m}$ thick, and then annealed at $423 \mathrm{~K}$ for $12 \mathrm{~h}$ after drying in vacuum at room temperature for 2 days. Three hundred sheets of the films on $\mathrm{Al}$ foils were rolled up and placed into a hollow cylindrical Al cell $14 \mathrm{~mm}$ in diameter and $45 \mathrm{~mm}$ high in order to get enough inelastic scattering intensity.

The inelastic neutron scattering measurements were performed with an inverted geometry time-of-flight (TOF) spectrometer LAM-40 [22] installed at the cold spallation neutron source in High Energy Accelerator Research Organization (KEK), Tsukuba, Japan. The energy resolution was about $200 \mu \mathrm{eV}$ at the elastic position on the energy gain side. The measurements were carried out at temperatures from $11 \mathrm{~K}$ to $300 \mathrm{~K}$, which is far below the bulk glass transition temperature $T_{g}(=373 \mathrm{~K})$, meaning that we mainly focused on the glassy state. At each temperature the TOF spectrum of the empty can including $300 \mathrm{Al}$ foils was carefully subtracted from that of the thin film after correcting for the counter efficiency, the self-shielding, and the incident neutron spectrum.

\section{RESULTS AND DISCUSSION}

The mean square displacement $\left\langle u^{2}\right\rangle$ can be evaluated from the $Q^{2}$ dependence of elastic intensity $I_{\mathrm{el}}(Q)$ using the equation $I_{\mathrm{el}}(Q) \sim \exp \left[-\left\langle u^{2}\right\rangle Q^{2}\right]$. As shown in previous papers $[17,18]$, the mean square displacement $\left\langle u^{2}\right\rangle$ decreases with film thickness for PS films with $M_{w}=2.90 \times 10^{5}$ and the thickness dependence was well described by the following equation:

$$
\left\langle u^{2}\right\rangle=\left\langle u^{2}\right\rangle_{\text {bulk }}\left|1-\left(\frac{d_{0}}{d}\right)^{\delta}\right|,
$$

where $\left\langle u^{2}\right\rangle_{\text {bulk }}, d, d_{0}$, and $\delta$ are mean square displacement of bulk, film thickness, constant, and exponent, respectively. Although we have no theoretical basis for this equation, we used it in analogy with the thickness dependence of $T_{g}$ suggested by Keddie et al. [1].

In this work we studied $\left\langle u^{2}\right\rangle$ for a film thickness of $400 \AA$ as a function of molecular weight $M_{w}$. First we focus on the temperature dependence of elastic intensity for three different molecular weights. Figure 1 indicates the temperature dependence of elastic intensity normalized to that of the lowest temperature for $M_{w}=2.90 \times 10^{5}, 1.05 \times 10^{6}$, and 1.88 $\times 10^{6}$ at $Q=2.20 \AA^{-1}$, and the error bars were evaluated from raw counts of the TOF spectrum at each temperature. The logarithm of the normalized elastic intensity decreases almost linearly with temperature as shown by solid lines in Fig. 1 in the low-temperature range, indicating that the vibrational motion is harmonic. In the case of bulk PS, onset of the so-called fast process is observed at around $200 \mathrm{~K}$, lead-

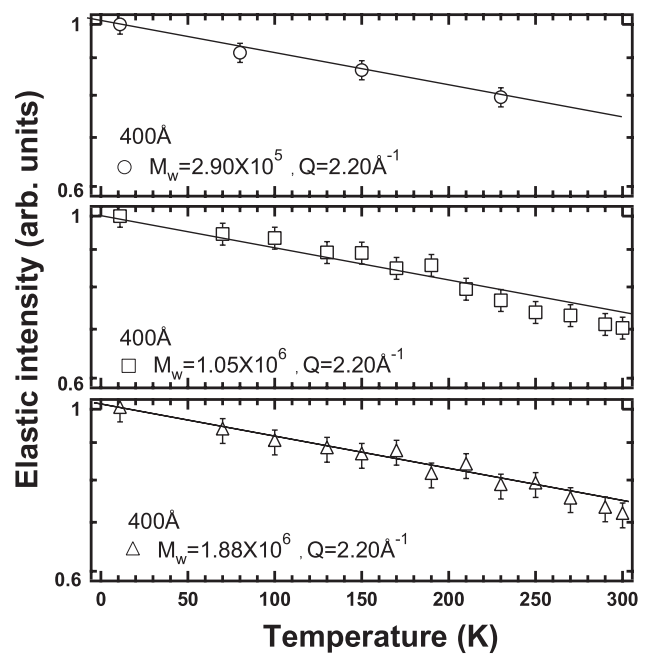

FIG. 1. Temperature dependence of the elastic scattering intensity divided by that of the lowest temperature $11 \mathrm{~K}$ for $M_{w}=2.90$ $\times 10^{5}(\bigcirc), 1.05 \times 10^{6}(\square)$, and $1.88 \times 10^{6}(\triangle)$ at $Q=2.20 \AA^{-1}$.

ing to a deviation from harmonic behavior [23,24]. In our former works, the onset temperature of the fast process increased ( $260 \mathrm{~K}$ for the $400 \AA$ film) and the spectra obtained were well scaled by a Bose population factor at $230 \mathrm{~K}$ for $400 \AA$ thin films $[17,18]$; hence, we concluded that the fast process was suppressed and hard to observe as the film thickness decreased. As seen in Fig. 1, the definite determination of onset temperature of the fast process was very difficult due to the large statistical errors. Hence, we do not know whether the fast process of thin films was suppressed or not clearly only from the data in this work, and we do not discuss the onset temperature of the fast process in this paper. The temperature dependences of the elastic intensity for three different $M_{w}$ 's were almost identical within the experimental error. We also evaluated $\left\langle u^{2}\right\rangle$ whose temperature dependence is shown in Fig. 2 for three different $M_{w}$ 's. The error bars in Fig. 2 were estimated from the uncertainties of

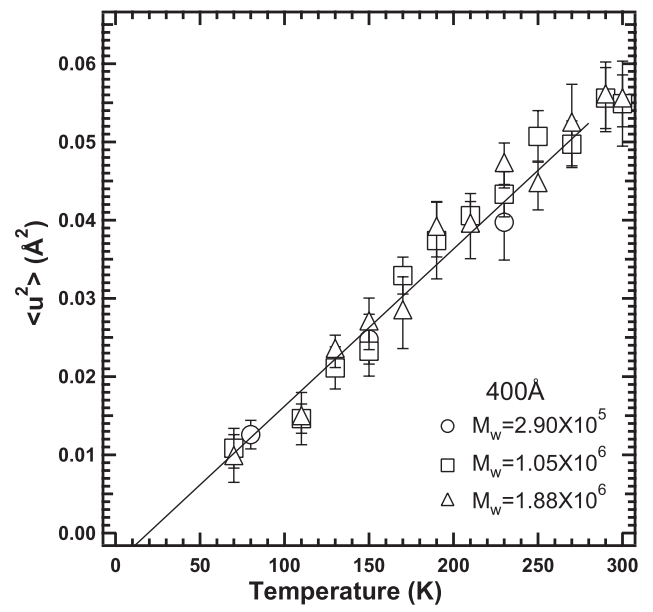

FIG. 2. Temperature dependence of the mean square displacement $\left\langle u^{2}\right\rangle$ for $M_{w}=2.90 \times 10^{5}(\bigcirc), 1.05 \times 10^{6}(\square)$, and $1.88 \times 10^{6}$ $(\triangle)$. The solid line is drawn to guide the eye. 


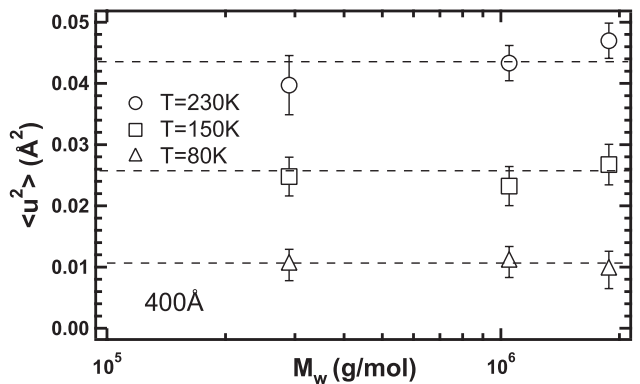

FIG. 3. $M_{w}$ dependence of the mean square displacement $\left\langle u^{2}\right\rangle$ at $80 \mathrm{~K}, 150 \mathrm{~K}$, and $230 \mathrm{~K}$ for $400 \AA$ thin films. The dotted line is drawn to guide the eye.

the fits. $\left\langle u^{2}\right\rangle$ is almost proportional to $T$ at low temperature as shown in Fig. 2, indicating again that the motion is harmonic. The evaluated values of $\left\langle u^{2}\right\rangle$ for different $M_{w}$ 's lie on a straight line within the experimental error. It was found that the values of $\left\langle u^{2}\right\rangle$ in the $400 \AA$ films are independent of $M_{w}$ in the temperature range examined. To confirm this, the values of $\left\langle u^{2}\right\rangle$ for the $400 \AA$ films are plotted as a function of $M_{w}$ at some temperatures in Fig. 3. It seemed that $\left\langle u^{2}\right\rangle$ slightly increased with $M_{w}$; however, this was caused by the large statistical errors and this tendency was not visible at other temperatures (see Fig. 2). If the confinement effects were dominant, a decrease of $\left\langle u^{2}\right\rangle$ would be observed with increasing $M_{w}$ because higher $M_{w}$ is more spatially confined than lower $M_{w}$ under the same film thickness. It was therefore concluded that the confinement effect was not the main reason for the decrease of mobility with film thickness.

Unfortunately we have to admit that the experimental error is not so small because of the very weak scattering intensity from the thin films in the present measurement. If the $M_{w}$ dependence of $\left\langle u^{2}\right\rangle$ were so small that it falls within the error bounds, we could not conclude that the decrease of $\left\langle u^{2}\right\rangle$ is caused by the interface layer. To check it, we plotted $\left\langle u^{2}\right\rangle$ as a function of the ratio of film thickness to twice of radius of gyration $\left(d / 2 R_{g}\right)$ in Fig. 4(a), which can be regarded as a measure of the deformation of polymer coils [25]. In the figure $\left\langle u^{2}\right\rangle$ 's for different film thicknesses $[17,18]$ are included. We also plotted the same data as a function of the film thickness $d$ for different molecular weights $M_{w}$ in Fig. 4(b). It is very clear that the $\left\langle u^{2}\right\rangle$ is scaled by $d$, not $d / 2 R_{g}$, within the accuracy in the measurements. This directly suggests that the $\left\langle u^{2}\right\rangle$ is not dominated by the deformation of polystyrene coils. It is concluded that the main reason for the decrease of $\left\langle u^{2}\right\rangle$ with film thickness is not the confinement of polymer coils, but the interface hard layer.

Next we have to consider the origin of the hard interface layer. Polymer chains tend to form ordered structure exhibiting layering [26] near the interface that is related to the radius of gyration of the polymer [27] as pointed out by Mukherjee et al. [28]. Zhang et al. [29] revealed orientation
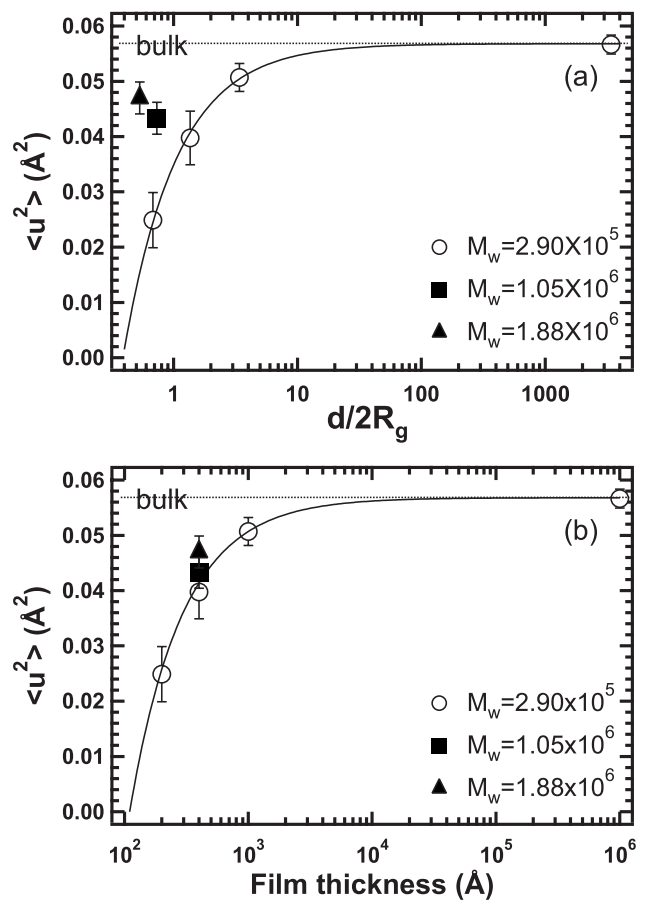

FIG. 4. (a) Mean square displacement $\left\langle u^{2}\right\rangle$ as a function of the ratio of the film thickness to twice the radius of gyration $\left(d / 2 R_{g}\right)$ at $230 \mathrm{~K}$ for $M_{w}=2.90 \times 10^{5}(\bigcirc), 1.05 \times 10^{6}(\boldsymbol{\square})$, and $1.88 \times 10^{6}(\mathbf{\Delta})$. (b) Mean square displacement $\left\langle u^{2}\right\rangle$ as a function of film thickness $(d)$ at $230 \mathrm{~K}$ for $M_{w}=2.90 \times 10^{5}(\bigcirc), 1.05 \times 10^{6}(\mathbf{\square})$, and 1.88 $\times 10^{6}(\mathbf{\Delta})$. Solid lines are the results of the fit with Eq. (1).

of molecular chain axis of poly(methyl methacrylate) at the interface using reflection-absorption infrared (RAIR) and surface-enhanced Raman scattering (SERS). Especially in the case of PS, phenyl rings tend to orient parallel to the interface between PS and substrate, revealed by IR-visible sum-frequency generation (SFG) spectroscopy [30]. Such orientation or ordering at the interface between polymer and substrate may be related to the hard layer, the so-called "dead layer." As a result of orientation or ordering at the interface, the mobility would be smaller than that in bulk due to the high contribution from the hard layer.

\section{CONCLUSION}

We have investigated the effect of molecular weight $\left(M_{w}\right)$ on the mean square displacement $\left\langle u^{2}\right\rangle$ in order to disclose the origin of the decrease of $\left\langle u^{2}\right\rangle$ with film thickness. The observed $\left\langle u^{2}\right\rangle$ values are independent of the molecular weight $M_{w}$ within the experimental error, and the molecular weight dependence of $\left\langle u^{2}\right\rangle$ expected from the confinement effect is much larger than the experimental error. It was therefore concluded that the decrease of $\left\langle u^{2}\right\rangle$ with film thickness was caused by the interface hard layer. 
[1] J. L. Keddie, R. A. L. Jones, and R. A. Cory, Europhys. Lett. 27, 59 (1994).

[2] J. L. Keddie, R. A. L. Jones, and R. A. Cory, Faraday Discuss. 98, 219 (1994)

[3] S. Kawana and R. A. L. Jones, Phys. Rev. E 63, 021501 (2001).

[4] J. A. Forrest, K. Dalnoki-Veress, J. R. Stevens, and J. R. Dutcher, Phys. Rev. Lett. 77, 2002 (1996).

[5] K. Fukao and Y. Miyamoto, Europhys. Lett. 46, 649 (1999).

[6] K. Fukao and Y. Miyamoto, Phys. Rev. E 64, 011803 (2001).

[7] K. Fukao and Y. Miyamoto, Phys. Rev. E 61, 1743 (2000).

[8] T. Kajiyama, K. Tanaka, and A. Takahara, Polymer 39, 4665 (1998).

[9] T. Kajiyama, K. Tanaka, N. Satomi, and A. Takahara, Macromolecules 31, 5150 (1998).

[10] N. Satomi, A. Takahara, and T. Kajiyama, Macromolecules 32, 4474 (1999).

[11] N. Satomi, K. Tanaka, A. Takahara, T. Kajiyama, T. Ishizone, and S. Nakahama, Macromolecules 34, 8761 (2001).

[12] H. Kim, A. Rühm, L. B. Lurio, J. K. Basu, J. Lal, D. Lumma, S. G. J. Mochrie, and S. K. Sinha, Phys. Rev. Lett. 90, 068302 (2003).

[13] K. Akabori, K. Tanaka, T. Nagamura, A. Takahara, T. Kajiyama, Macromolecules 38, 9735 (2005).

[14] C. L. Soles, J. F. Douglas, W.-1. Wu, and R. M. Dimeo, Phys. Rev. Lett. 88, 037401 (2002).

[15] C. L. Soles, J. F. Douglas, W. Wu, H. Peng, and D. W. Gidley, Macromolecules 37, 2890 (2004).

[16] B. Frick, K. Dalenoki-Veress, J. A. Forrest, J. Dutcher, C. Murray, and A. Higgins, Eur. Phys. J. E 12, (s01), 93 (2003).
[17] R. Inoue, T. Kanaya, K. Nishida, I. Tsukushi, and K. Shibata, Phys. Rev. E 74, 021801 (2006).

[18] R. Inoue, T. Kanaya, K. Nishida, I. Tsukushi, and K. Shibata, Phys. Rev. Lett. 95, 056102 (2005).

[19] J. A. Forrest, C. Svanberg, K. Révész, M. Rodahl, L. M. Torell, and B. Kasemo, Phys. Rev. E 58, R1226 (1998).

[20] T. Miyazaki, K. Nishida, and T. Kanaya, Phys. Rev. E 69, 061803 (2004).

[21] J. Brandrup, E. H. Immergut, and E. A. Grulke, Polymer Handbook (Wiley, New York, 1998).

[22] K. Inoue, Y. Ishikawa, N. Watanabe, K. Kaji, Y. Kiyanagi, H. Iwasa, and M. Kohgi, Nucl. Instrum. Methods Phys. Res. A 238, 401 (1985).

[23] B. Frick, U. Buchenau, and D. Richter, Colloid Polym. Sci. 273, 413 (1995).

[24] T. Kanaya, T. Kawaguchi, and K. Kaji, J. Chem. Phys. 104, 3841 (1996).

[25] K. Shuto, Y. Oishi, T. Kajiyama, and C. C. Han, Macromolecules 26, 6589 (1993).

[26] J. Kraus, P. Müller-Buschbaum, T. Kuhlmann, D. W. Schubert, and M. Stamm, Europhys. Lett. 49, 210 (2000).

[27] M. K. Sanyal, J. K. Nasu, A. Datta, and S. Banerjee, Europhys. Lett. 36, 265 (1996).

[28] M. Mukherjee, M. Bhattacharya, M. K. Sanyal, T. Geue, J. Grenzer, and U. Pietsch, Phys. Rev. E 66, 061801 (2002).

[29] J. M. Zhang, D. H. Zhang, and D. Y. Shen, Macromolecules 35, 5140 (2002).

[30] K. S. Gautam, A. D. Schwab, A. Dhinojwala, D. Zhang, S. M. Dougal, and M. S. Yeganeh, Phys. Rev. Lett. 85, 3854 (2000). 